\title{
Los estudiantes universitarios: vulnerabilidad, atención e intervención en su desarrollo
}

\author{
Angélica Riveros Rosas
}

\begin{abstract}
Resumen
La salud física y mental de los estudiantes de universidades públicas requiere atención especial, pues está estrechamente vinculada con las actividades académicas que llevan a cabo para formarse como profesionistas. Los estudiantes con más dificultades psicológicas corren mayores riesgos durante la carrera ya que pueden tomar decisiones poco afortunadas que afecten el resto de su vida adulta. Además, los diversos tipos de carreras en las universidades públicas implican perfiles particulares. En el caso de los estudiantes de carreras profesionalizantes, se espera que los estudiantes trabajen al tiempo que estudian y que obtengan éxito personal y económico rápidamente. El presente estudio presenta los hallazgos sobre la afectación en estudiantes de alto y bajo desempeño académico, así como resultados de programas de intervención en el Centro de Orientación Educativa y Psicológica de la Facultad de Contaduría y Administración de la Universidad Nacional Autónoma de México (UNAM). Se destaca la importancia de contar con opciones al interior de las facultades y de la amplitud de los efectos de mejorar la salud mental de los estudiantes universitarios.
\end{abstract}

Palabras clave: bienestar, salud mental, intervenciones, estudiantes universitarios.

\section{UNIVERSITY STUDENTS: VULNERABILITIES, WELLBEING AND INTERVENTION}

\begin{abstract}
The mental health of students in public universities requires especial attention because it interacts closely with academic activities which are indispensible for sucessful professional training. Students with more psychological difficulties are at higher risk during their studies since they could make delicate, unfortunate decisions that may affect most of their adult lives. In addition, different university careers represent relatively specific profiles. Some careers are normally expected to lead to employment even before graduation. These students are also expected to succeed quickly both personally and in terms of income. The present paper describes recent findings from a counseling center in a large public university, on psychological difficulties as related to specific aspects of academic performance, as well as the effects of some interventions. Results are discussed in terms of the need for improved organizational options within university professional schools in the context of improving students' mental health.
\end{abstract}

Key words: wellbeing, mental health, interventions, university students. 


\section{Angélica Riveros Rosas}

ariveros@fca.unam.mx

Facultad de Contaduría y Administración, UNAM. Cursó sus estudios en Psicología desde licenciatura hasta doctorado en la UNAM en el área de salud. Es tutor principal de maestría y doctorado en Psicología y Ciencias de la Administración. En su producción destaca la evaluación de intervenciones para mejorar la calidad de vida, la salud y la interacción interpersonal. Sus líneas de investigación incluyen estrés en escenarios académicos y de atención a la salud.

\section{Agradecimientos}

La autora agradece al equipo del Centro de Orientación Educativa por su trabajo dedicado en el funcionamiento del Centro. También a los miembros de la Secretaría de Relaciones y Extensión Universitaria por su apoyo. Finalmente se agradece a los alumnos por su confianza en el trabajo del Centro.

Los estudiantes que ingresan a las universidades públicas se caracterizan por su heterogeneidad, pues las condiciones en las que inician su formación profesional son muy diferentes. Sus habilidades de estudio, promedio de calificaciones e interés en las diferentes materias son fácilmente reconocibles por los docentes y pueden llevarlos a tratar a los alumnos en concordancia, mostrando interés y apoyo a partir de cómo perciben al estudiante. Si bien esto es de espe-

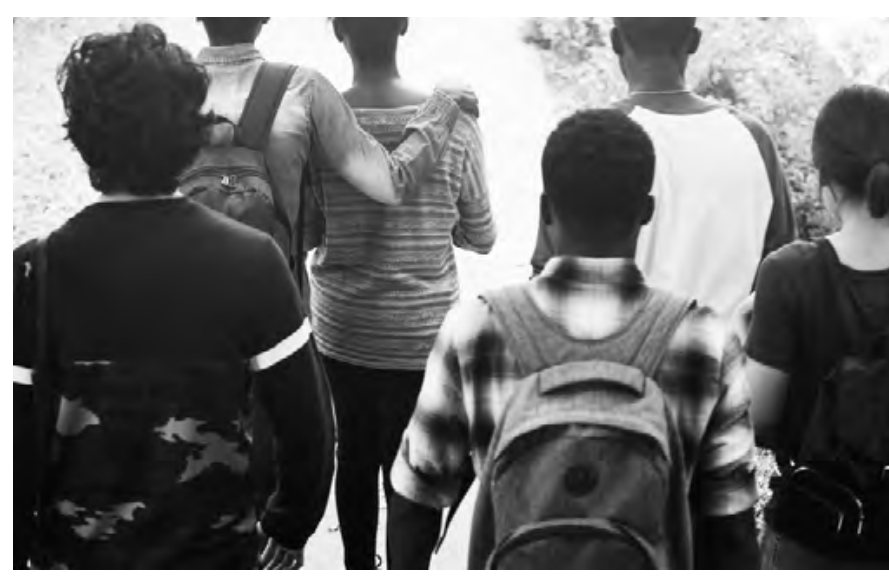
rarse, puede haber efectos particulares hacia los extremos, es decir, alumnos consistentemente percibidos como 'buenos' o 'malos'. Es relativamente fácil concluir que los más destacados son talentosos y merecedores de las mejores oportunidades y el éxito, mientras que los menos, tendrían limitaciones que hacen que sus aspiraciones no sólo deban ser más modestas, sino es que podrían estar relativamente 'condenados al fracaso', al menos respecto a ciertas materias clave.

Los estudiantes inician su tránsito académico en la universidad desde puntos muy diversos, cada uno tiene antecedentes personales, familiares y de bienestar que pueden propiciar brechas en su formación. La relación entre desempeño, 
1. La evitación se refiere a una tendencia relativamente estable a responder alejándose o retirándose de aquello que se considera desagradable o para lo que no se cuenta con los recursos para su manejo, en este caso situaciones o tareas en las que pueda fallar. salud física y salud mental en este periodo delinea y consolida mucho de su funcionamiento y expectativas en la vida adulta. Así, el reconocimiento cada vez más puntual de cómo interactúa la salud con el funcionamiento estudiantil posibilita incidir en la mejora de las condiciones por las que transitan en su formación.

Algunos problemas frecuentes de los alumnos incluyen dificultades de planeación y organización de tareas, intolerancia a la incertidumbre y evitación ${ }^{1}$ ante el fracaso (Martin, 2008). Estas dificultades pueden expresarse como una tendencia a postergar actividades que parecen poco agradables, o bien demasiado importantes como para iniciarlas sin estar 'inspirados', o 'en su mejor momento' y dar prioridad a actividades distractoras, más atractivas o estimulantes como las de redes sociales o la televisión, entre muchas otras. Distraerse disminuye en lo inmediato la angustia de un pendiente académico desagradable o, si son perfeccionistas, no toman decisiones por temor a decepcionar. También suelen esperar la activación de la angustia de la entrega inminente porque creen 'trabajar mejor bajo presión' (Guzman, 2013). Este tipo de procrastinación entre estudiantes universitarios suele oscilar entre $70 \%$ al 95\% para actividades como escribir, estudiar o hablar con profesores, y del 25\% (en su forma más incapacitante) en tareas importantes, llevando incluso al auto sabotaje (Klassen, Krawchuk y Rajani, 2007). Además de generar estrés sostenido y malos resultados, estas conductas propician convicciones como incapacidad para autorregularse, planificar, ser eficaz, así como baja valía personal. A su vez, esto favorece estados emocionales desagradables y en ocasiones, incapacitantes relativamente permanentes.

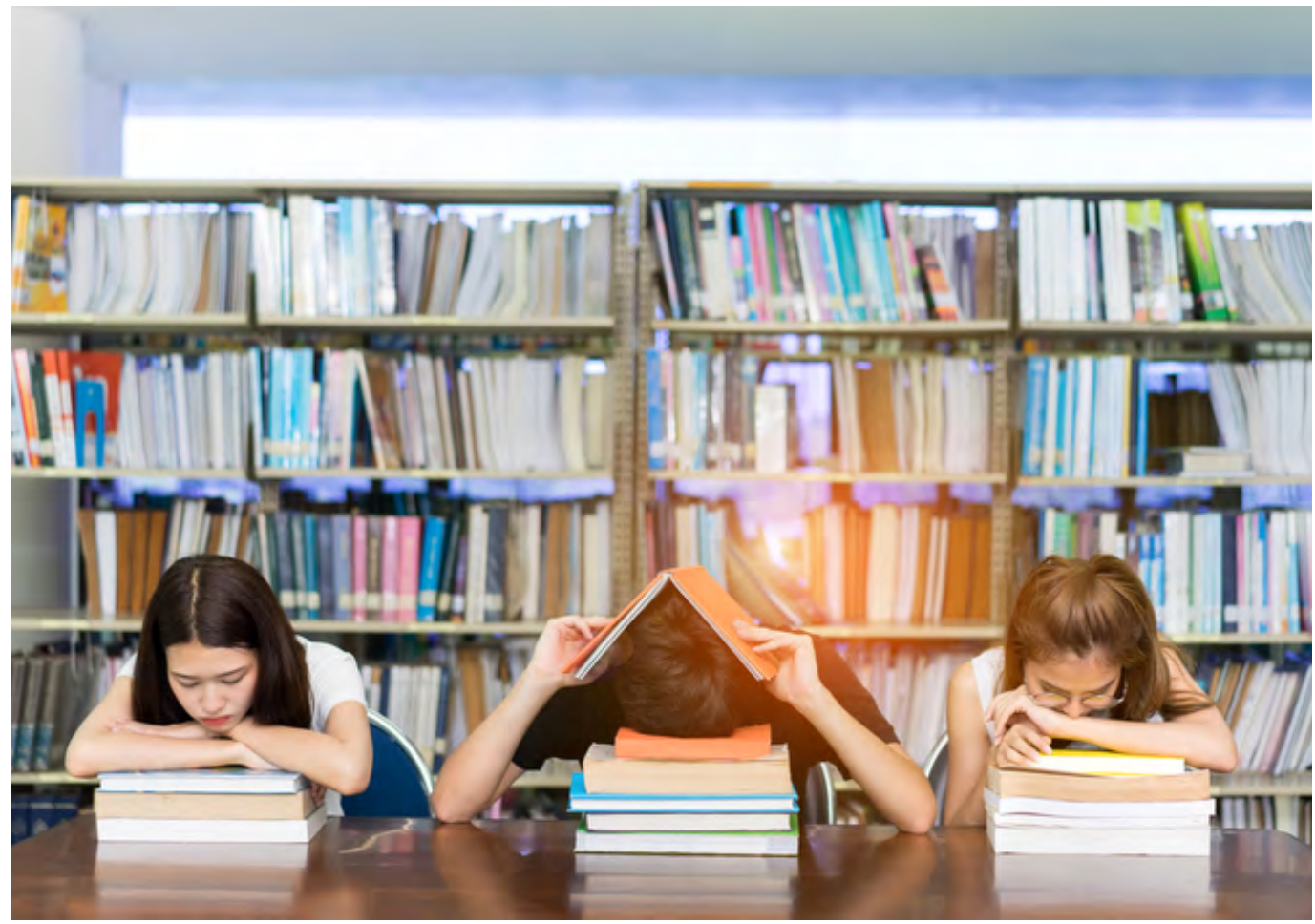


En sentido psicofisiológico, las emociones negativas como la ansiedad, el enojo y la tristeza impactan el desempeño, pues activan en el cerebro áreas relacionadas con atención excesiva al malestar y el recuerdo de situaciones congruentes con ese estado emocional. También se activan áreas relacionadas con la evitación y disminuye la actividad en áreas relacionadas con tomar decisiones, concentrarse y crear afiliaciones y afectos positivos (Singer y Klimecki, 2014). Así, se ven afectadas las expectativas sobre sí mismos, tales como lo que pueden tolerar, lo que los mantiene tranquilos, su capacidad para autorregularse y para aprender. Por ejemplo, la hipersensibilidad al rechazo facilita estilos de interacción caracterizados por timidez, sumisión, inhibición emocional y explosiones emocionales de ira, angustia, tristeza, o impulsividad, así como recuerdos relacionados con esos estados de ánimo. A mediano plazo y de manera indirecta, pero notoria, esto afecta cómo se conciben como estudiantes y en las relaciones con sus pares, el apoyo social que perciben y sus relaciones de pareja. Finalmente, se ha documentado que estados prolongados de este tipo también ocasionan desgaste físico, agotamiento y pueden contribuir a una propensión a enfermedades como arteroesclerosis, infecciones recurrentes, envejecimiento prematuro del corazón o riesgo cardiovascular (Denollet, 2013). En síntesis, la investigación científica muestra de manera cada vez más contundente que la salud física y la mental interactúan constantemente.

Los efectos del malestar emocional son muy variados, la ansiedad es más intensa en estudiantes con bajo promedio (Macías y Hernández, 2008). En cursos de matemáticas o finanzas, las mujeres sienten más ansiedad ante clases y exámenes, aunque en la realidad reprueban menos y tiendan a lograr calificaciones más altas (Zainal, Alwf y Jaafar, 2010). Sin embargo, en general, las consecuencias son muy similares: los alumnos que viven su formación con malestar tienden a evitar esos aspectos del conocimiento en su vida profesional independientemente de su desempeño académico. Finalmente, hay expectativas familiares, sociales y profesionales que también afectan el bienestar del estudiante.

Las carreras profesionalizantes se caracterizan por incluir actividades de servicio requeridas por la sociedad más que actividades de investigación y enseñanza. Carreras como Contaduría y Administración incluyen condiciones como una inserción temprana en la vida laboral, generalmente durante la segunda mitad de la carrera y hay expectativas de

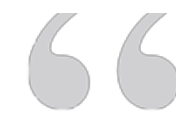

Hay estudiantes que pueden llevar años sobrellevando formas importantes de malestar como depresión, ansiedad, problemas de enojo o de autoestima... 
Figura 1.

Diferencias entre alumnos con promedio alto y bajo del bachillerato en indicadores de bienestar emocional al ingreso a la carrera
Figura 2.

Diferencia entre alumnos con promedio alto y bajo del bachillerato en características de relación de pareja al ingreso a la carrera disciplina y éxito económico y social a corto plazo. Las diferencias en bienestar y desempeño son detectables desde su ingreso. Un estudio con 1361 estudiantes de las carreras de Contaduría, Administración e Informática de la Facultad de Contaduría y Administración de la UNAM, muestra que las diferencias más importantes entre alumnos de alto y bajo promedio de bachillerato son la procrastinación y la agresividad, en general, los estudiantes con alto promedio muestran menor enojo, menor agresividad y menos adicciones (véase la Figura 1).

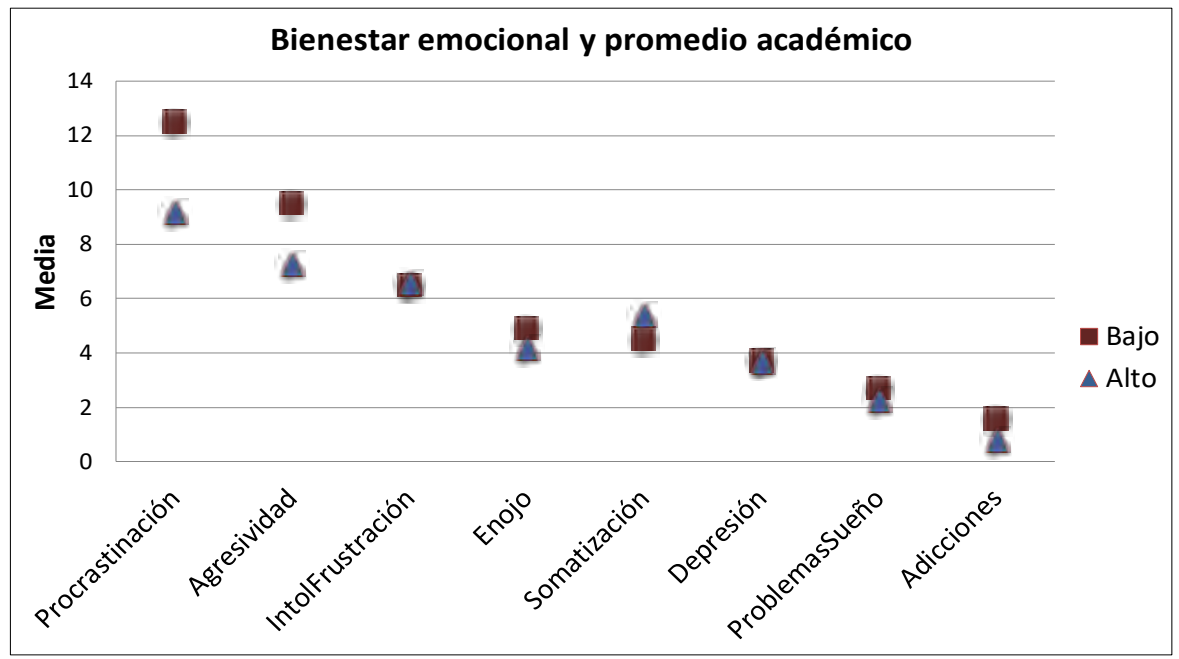

En el caso de la Facultad de Contaduría y Administración, inicialmente hay pocas diferencias en intolerancia a la frustración, síntomas de depresión o problemas de sueño, probablemente porque al principio de la carrera tiene algún papel el entusiasmo de haber sido admitido y empezar un nuevo ciclo. En cambio, las diferencias en las relaciones de pareja son muy marcadas. Los alumnos de bajo promedio han tenido relaciones menos estables, con prácticas vulnerantes como chantaje, distintas formas de violencia y sentirse forzados a actividades que no desean por miedo al abandono de la pareja (incluyendo las sexuales) (véase la Figura 2).

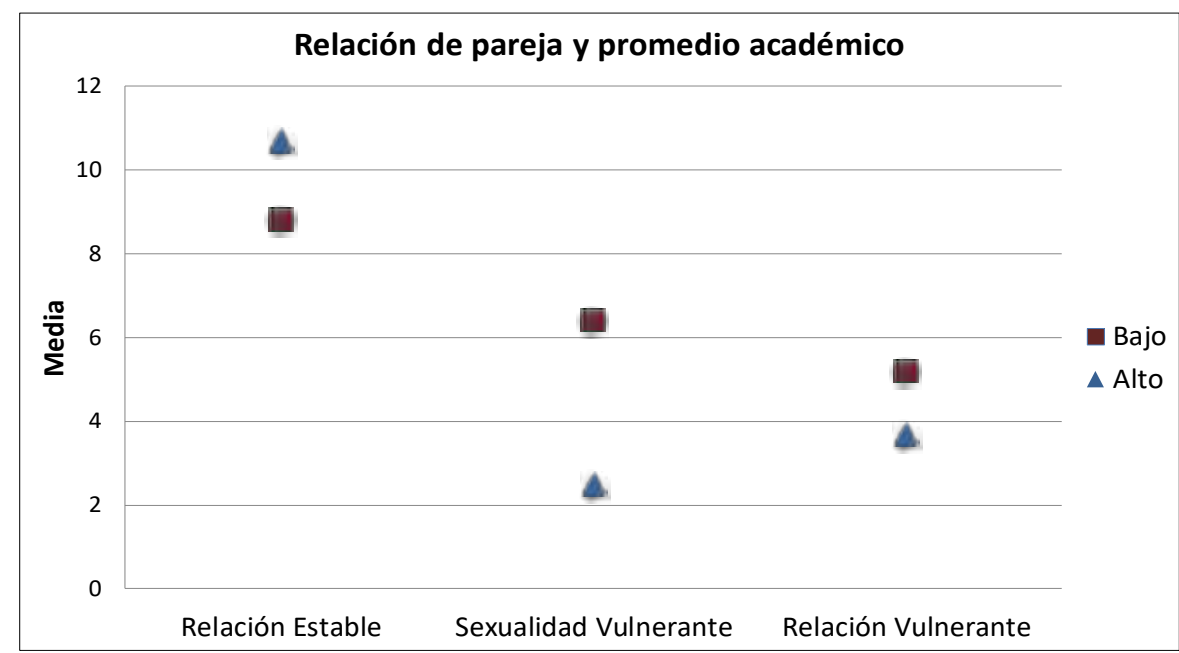


Figura 3.

Correlación entre grupos de alto y bajo promedio del bachillerato
Los alumnos con mayor promedio refieren mejor relación con los padres, hay mayor involucramiento de éstos en la importancia de sus estudios; en contraparte, los de bajo promedio refieren más violencia familiar durante su niñez. Así, es comprensible pero no necesariamente obvio que los estudiantes con bajo promedio ingresen más interferidos en sus actividades académicas y usen menos estrategias de estudio que les ayuden a cumplir con los requisitos puestos por el profesor.

Un análisis estadístico de función discriminante permite reconocer, entre diversas variables, cuáles distinguen grupos por algún factor, en este caso, las diferencias entre altos y bajos promedios y las características de bienestar psicosocial. LoS resultados revelaron diferencias en bienestar, procrastinación, sexualidad vulnerante, agresividad y pocas relaciones sociales positivas. Las relativas al estudio no fueron las más fuertes en el análisis y sólo entraron en la función distracción durante el estudio y las estrategias dirigidas al cumplimiento escolar (véase la Figura 3).

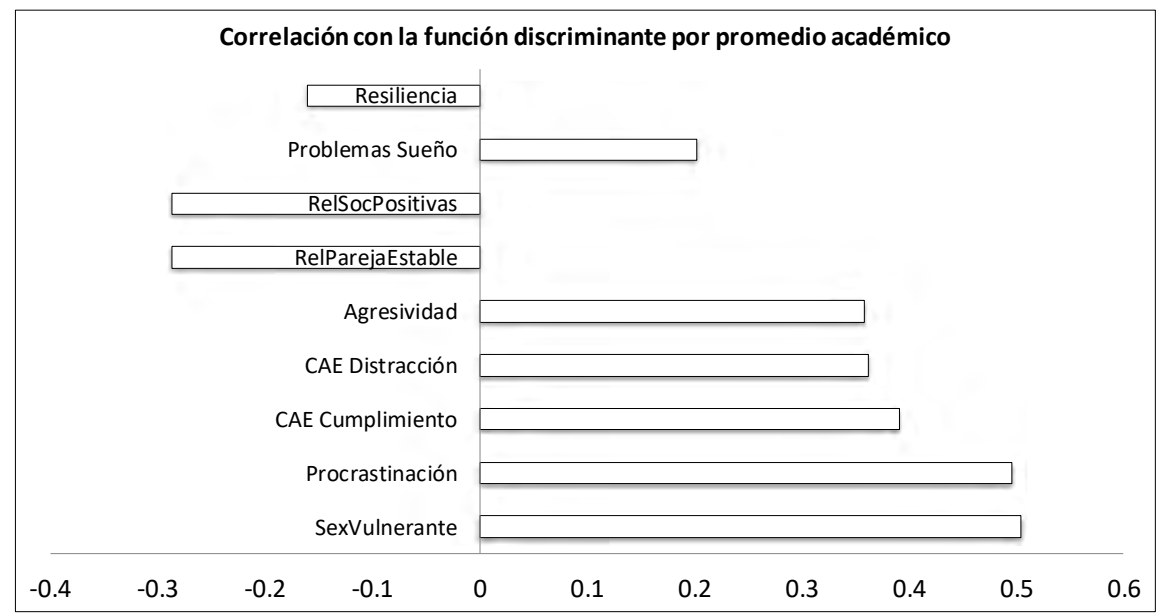

La figura anterior muestra, de mayor a menor, la asociación con las características que mejor distinguen a los grupos por promedio (función discriminante). Las asociaciones más fuertes fueron sexualidad vulnerante y procrastinación, y las más débiles resiliencia o capacidad para recuperarse ante situaciones adversas. Las relaciones sociales positivas, resiliencia y relación de pareja estable, fueron las únicas que mostraron asociación inversa y qué funcionan como características protectoras de la salud mental. El resto funcionan como características vulnerantes.

La procrastinación es una característica conocida por su efecto en el funcionamiento estudiantil. Al examinar los factores predictores de la procrastinación destacaron la intolerancia a la frustración, el malestar ante situaciones sociales y la agresividad en todos los alumnos (véase la figura 4). Este análisis permite identificar sólo aquellas que predicen la procrastinación. La R² muestra el porcentaje de contribución de la procrastinación que es explicable por las características estudiadas, en la figura 4 se muestran ordenadas por su contribución a dicho porcentaje. La beta muestra la carga que tiene cada característica y permite reconocer la dirección que tiene en relación a la variable predicha. 
Figura 4.

Predicción de procrastinación

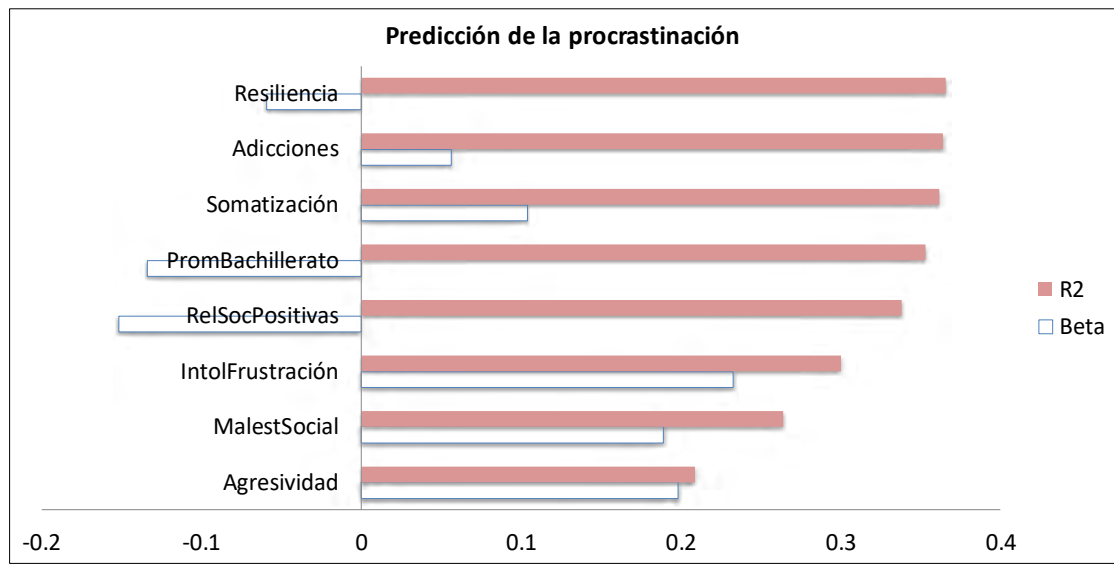

La agresividad fue la variable que más explica la procrastinación, seguida de malestar social e intolerancia a la frustración. Las que menos contribuyeron fueron resiliencia y adicciones. Nuevamente se observa que la mayor predicción es por características vulnerantes, mientras que las protectoras tuvieron signos negativos en la predicción de la procrastinación.

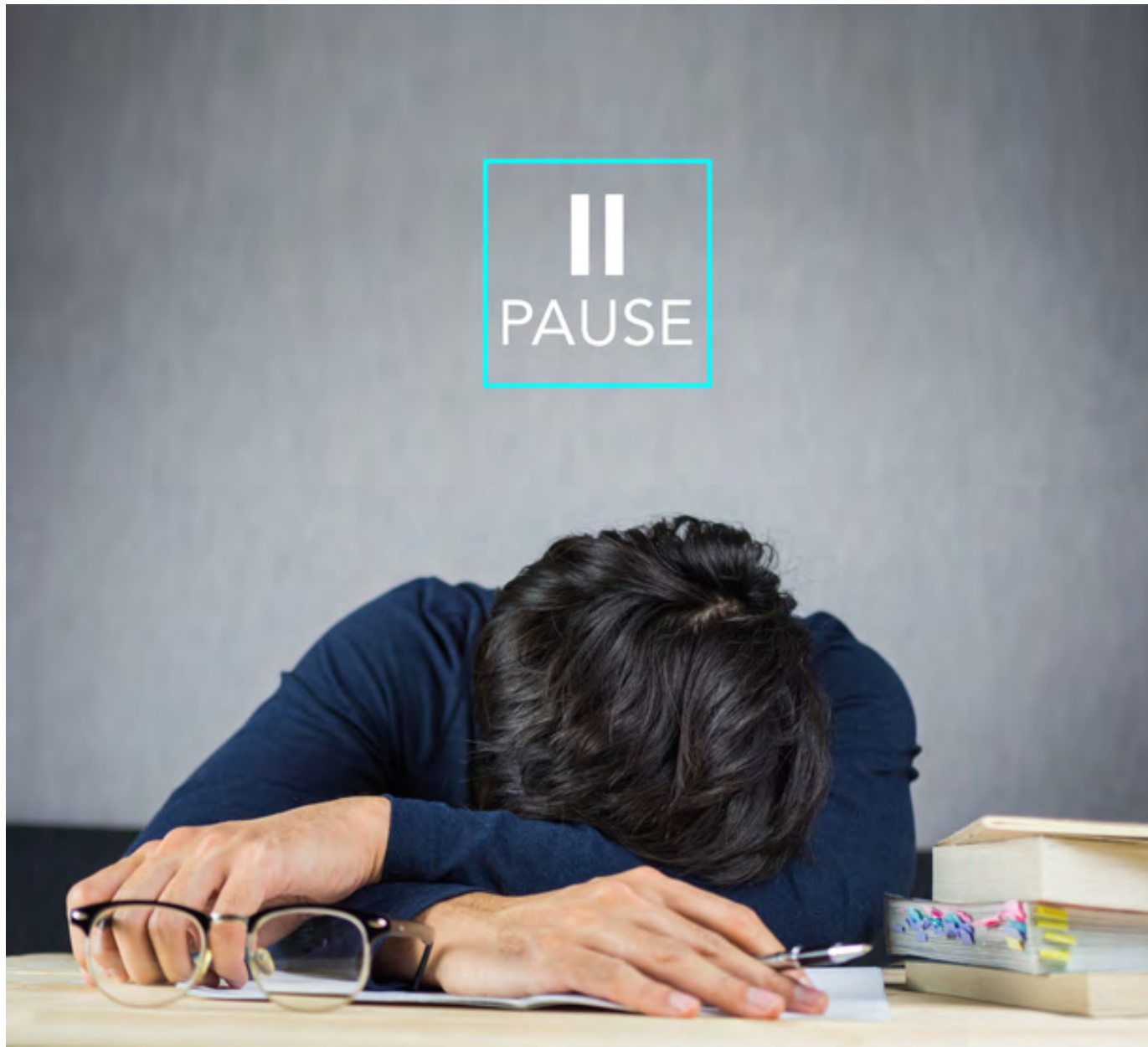


Estos resultados muestran la compleja relación entre desempeño y salud mental congruentes con otros resultados más a largo plazo. La capacidad percibida de autorregularse predice procrastinación y demora en la entrega de trabajos, autosabotaje (distracción a sabiendas de que será contraproducente), ansiedad ante exámenes y resultados por debajo de lo esperable (Klassen et al., 2007). En lo general, también se ha documentado que las dificultades de salud mental son las que generan más pérdida de días laborables, destacando la depresión, la fobia social y el trastorno de estrés postraumático. De hecho, todo parece indicar que, por ejemplo, se pierden más días laborables por depresión que por enfermedades crónicas (Lara, Medina, Borges y Zambrano, 2007). Para acabar de complicar este contexto, el debut de los problemas de salud mental prevalentes en México como depresión, trastornos de ansiedad y adicciones predomina entre los 18 y 27 años de edad (Medina-Mora et al., 2003), lo cual incluye claramente el periodo de la vida universitaria e incide temprano en estos problemas que, idealmente, se requeriría prevenir su aparición.

\section{El papel de los centros de atención en la UNAM, el caso del COEFCA}

Los centros de atención encargados de preservar y recuperar el bienestar de los estudiantes en las facultades universitarias representan ventajas importantes: facilitan acudir a ellos porque están en el sitio mismo de sus principales actividades cotidianas, facilitan el reconocimiento de las necesidades particulares de su profesión, sus servicios están adecuados a sus necesidades y permiten aprendizaje bajo supervisión.

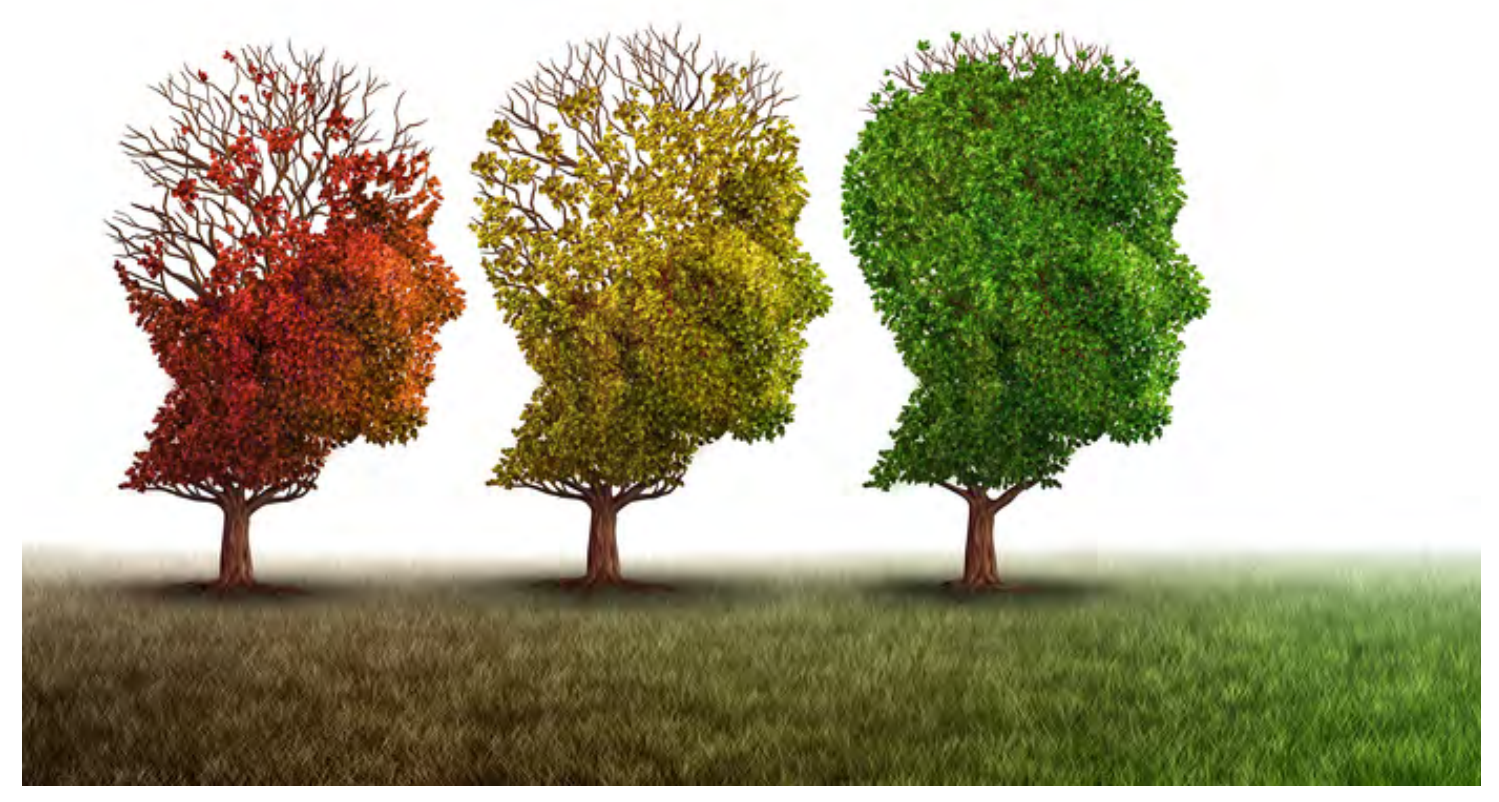


Algunos antecedentes institucionales incluyen a los centros de formación y servicios psicológicos de la Facultad de Psicología (ir al sitio), los centros de la Dirección General de Orientación y Atención Educativa y a los de atención a la salud mental de la Facultad de Medicina (ir al sitio), por mencionar algunos.

En el caso particular del Centro de Orientación Educativa y Psicológica de la Facultad de Contaduría y Administración (COEFCA) se busca:

A. Coadyuvar a la mejora constante y formación integral de los miembros de la comunidad estudiantil;

B. Favorecer el desarrollo armónico en el contexto de las actividades que comprenden su formación como profesionistas;

C. Favorecer su desarrollo como individuos plenos, congruentes, responsables y respetuosos de sí mismos y de sus profesiones (que eventualmente deberán representar de manera digna).

Entre otros, también cuenta con programas para el manejo de conflicto en el aula o alumnos con necesidades especiales. En el caso de apoyo a dificultades académicas específicas, se recurre a la colaboración de pares destacados en dichas materias y profesores voluntarios, desarrollo de materiales de evaluación psicosocial, impartición de talleres y canalización de casos que requieren atención más especializada. Todas las actividades del COEFCA asumen que el aprendizaje es necesario para el cambio duradero en los comportamientos y el alcance de metas.

El COEFCA abrió sus puertas en 2010 con la idea de ofrecer a los alumnos un espacio que, vinculando actividades de atención, formación e investigación, permita la atención a sus necesidades estudiantiles de manera más amplia. Así, se inserta dentro de la Secretaría de Relaciones y Extensión Universitaria que concentra servicios como bolsa de trabajo, actividades deportivas y culturales y servicio social.

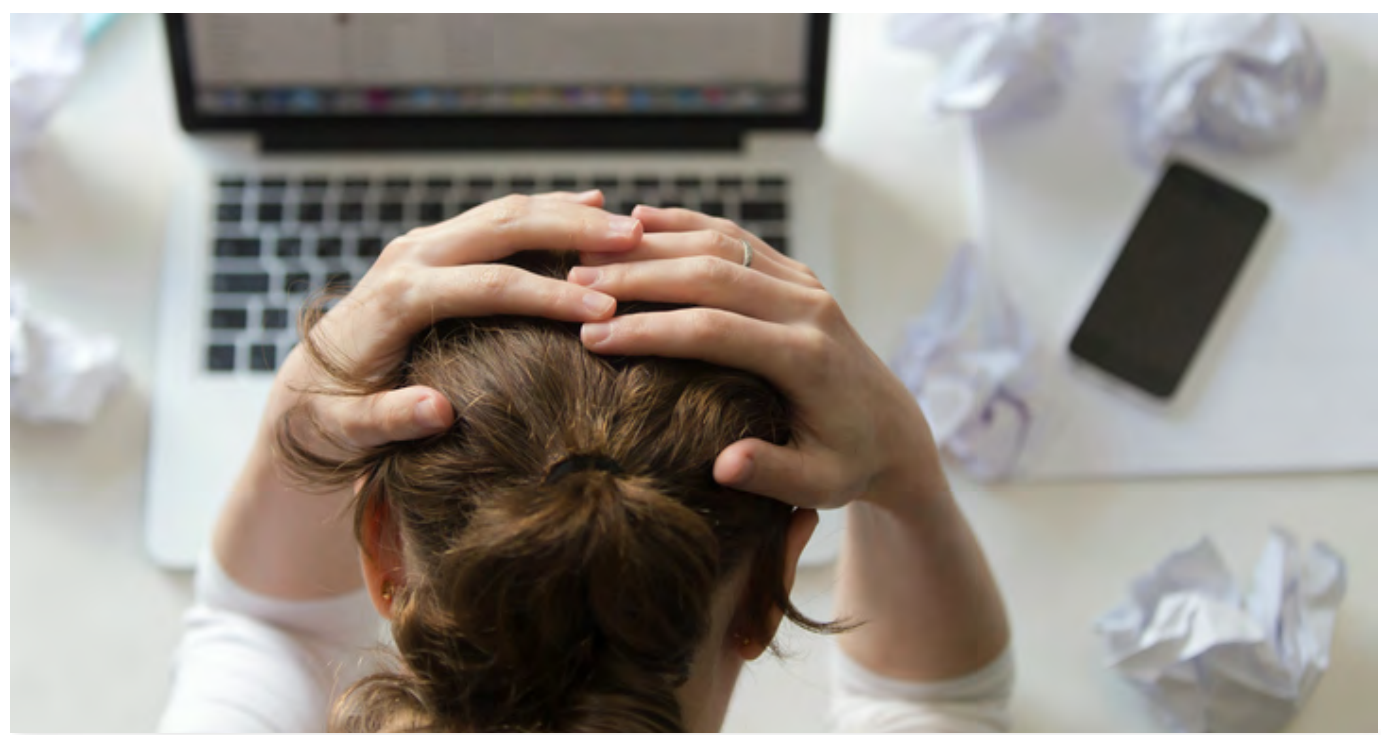


Los contenidos académicos que más suelen requerir servicios, especialmente cerca del final del semestre se relacionan con las matemáticas: Estadística, Contabilidad, Costos, Finanzas y Matemáticas. Las evaluaciones psicosociales han permitido desarrollar y adaptar talleres a las condiciones de estudiantes que trabajan, con atención puntual y breve, con contenidos sucintos y en horarios accesibles.

Las evaluaciones sobre bienestar indican necesidades que pueden atenderse mediante talleres, los cuatro más requeridos incluyen: Autoestima y asertividad, Autocontrol de emociones, Habilidades de interacción y Estrategias de estudio. La mayor captación para éstos ocurre al principio del semestre, y los más solicitados son Autoestima y asertividad y Autocontrol de emociones.

La modalidad de atención personalizada es una de las más solicitadas, principalmente por dificultades con el manejo de emociones (tristeza, enojo y

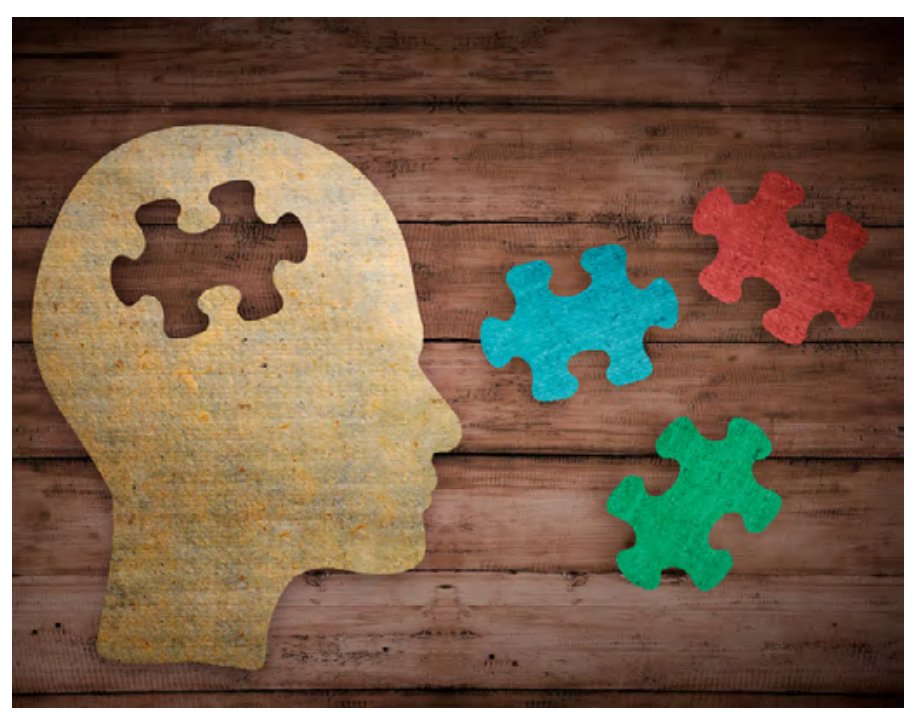
espectro ansioso), la afectación en autoestima y autoconcepto, las dificultades de interacción con pares y profesores, y los problemas de salud y somatización. Estos problemas afectan diversas esferas de la vida del alumno y las más frecuentes son académicas, de familia y de pareja. En general, como ocurre en otros países, los problemas por lo que se solicita atención representan al momento de hacerlo, empeoramiento importante o crisis. La necesidad de atención disminuye conforme pasa la intensidad de la crisis y, con alguna frecuencia, parece bastar haber solicitado la ayuda, aunque hay casos que después de hacer la solicitud ya no se presentan (Olfson et al., 2009).

Hay estudiantes que pueden llevar años sobrellevando formas importantes de malestar como depresión, ansiedad, problemas de enojo o de autoestima, que se benefician de manera importante de recibir la atención de estos centros. En el COEFCA se ha observado que, por ejemplo, los niveles de ansiedad, depresión o agresión de la población atendida bajan de niveles clínicos a moderados, a mínimos o sin ansiedad o depresión. Después de la atención los estudiantes refieren mayor tolerancia y cercanía con su familia, mejor capacidad de expresión, capacidad para permanecer en situaciones difíciles o incómodas. En la esfera académica refieren mejor organización de su tiempo, tolerancia al error durante el aprendizaje, delimitación de responsabilidades que mejora la interacción y el trabajo en equipo. En salud física refieren mejor cuidado de su alimentación, actividad física, habilidades para tranquilizarse, 
reducción o eliminación de hábitos que perjudican su salud, y capacidad para la aceptación y cuidado de su cuerpo.

Son muchos los retos que se enfrentan durante la formación universitaria, los estudiantes requieren de las mejores condiciones posibles para su desarrollo y los profesores para su labor académica. El no prestar atención a las necesidades académicas, sociales y personales genera riesgos sobre limitaciones autoimpuestas, al acceso a mejores condiciones para aprender e incluso problemas de violencia o dificultades legales.

La participación de expertos y de toda la comunidad en la búsqueda de una Universidad segura y promotora del desarrollo requiere de la atención a todos, pero especialmente hacia los miembros más vulnerables en la construcción de una sociedad cada vez más justa.

\section{Referencias}

- Denollet, J. (2013). Interpersonal sensitivity, social inhibition, and type D personality: How and when are they associated with health?. En Marin y Miller, Psychological Bulletin, 139(5), 991-997. Recuperado de: http://www.cell.com/current-biology/ fulltext/S0960-9822(14)00770-2.

* Lara, M. C., Medina Mora, M. E., Borges, G., y Zambrano, J. (2007). Social cost of mental disorders: Disability and work days lost. Results from the Mexican Survey of Psychiatric epidemiology. Salud Mental, 30(5), 4-11. Recuperado de: http://www. redalyc.org/articulo.oa?id=58230502.

* Guzmán P., David (2013). Procrastinación. Una mirada clínica (pp. 2-33). Tesis de Máster de Psicología Clínica y de la Salud. Barcelona: Instituto Superior de Estudios Psicológicos. https://drive.google.com/file/d/OB Rzkq9hb1dfWWxZTWowNmYwbnc/view.

- Klassen, R. M., Krawchuk, L. L. y Rajani, S. (2007). Academic procrastination of undergraduates: Low self-efficacy to self-regulate predicts higher levels of procrastination. Contemporary Educational Psychology, 33, 915-931. DOI: https://doi. org/10.1016/j.cedpsych.2007.07.001.

* Macías Martínez, D. y Hernández Pozo, M. R. (2008). Indicadores conductuales de ansiedad escolar en bachilleres en función de sus calificaciones en un examen de matemáticas. Universitas Psychologica, 7(3), 767-785. Recuperado de: http://revistas.javeriana.edu.co/index.php/revPsycho/article/view/391. 
* Martin, A. J. (2008). Enhancing student motivation and engagement: The effects of a multidimensional intervention, Contemporary educational psychology, 33(2), 239-269. DOI: https://doi.org/10.1016/j.cedpsych.2006.11.003.

* Medina-Mora, M. E., Borges, G., Lara, C., Benjet, C., Blanco, J., Flelz, C., Villatoro, J., Rojas, E., Zambrano, J., Casanova, L., y Aguilar-Gaxiola, S. (2003). Prevalencia de trastornos mentales y uso de servicios: resultados de la Encuesta Nacional de Epidemiología Psiquiátrica en México. Salud Mental, 26(4), 1-16.

Olfson, M., Mojtabai, R., Sampson, N. A., Hwang, I., Druss, B., Wang, P. S., Wells, K. B., Pincus, H. A., y Kessler, R. C. (2009). Dropout from outpatient mental health care in the United States. Psychiatric Services, 60(7), 898-907. DOl: https://doi. org/10.1176/ps.2009.60.7.898.

Singer, T. y Klimecki, O. (2014). Empaty and Compassion. Current Biology, 24(18). R875-R878. DOI: http://dx.doi.org/10.1016/j.cub.2014.06.054.

* Zainal, R., Alwf, F. y Jaafar, N. (2010). A case study on mathematics anxiety for mathematics of finance course. US-China Education Review, 7(11), 1-11. Recuperado de: https://eric.ed.gov/?id=ED514771.

\section{Cómo citar este artículo}

* Riveros Rosas, Angélica (2018). Los estudiantes universitarios: vulnerabilidad, atención e intervención en su desarrollo. Revista Digital Universitaria (RDU). Vol. 19, núm. 1 enero-febrero. DOI: http://doi.org/10.22201/codeic.16076079e.2018. v19n1.a6. 